\title{
Associations between postpartum depression and assistance with household tasks and childcare during the COVID-19 pandemic: evidence from American mothers
}

\author{
Theresa E. Gildner ${ }^{1 *}$, Glorieuse Uwizeye ${ }^{2,3}$, Rebecca L. Milner ${ }^{2}$, Grace C. Alston ${ }^{2}$ and Zaneta M. Thayer 2,4
}

\begin{abstract}
Background: The early postpartum period is recognized cross-culturally as being important for recovery, with new parents receiving increased levels of community support. However, COVID-19-related lockdown measures may have disrupted these support systems, with possible implications for mental health. Here, we use a cross-sectional analysis among individuals who gave birth at different stages of the pandemic to test (i) if instrumental support access in the form of help with household tasks, newborn care, and care for older children has varied temporally across the pandemic, and (ii) whether access to these forms of instrumental support is associated with lower postpartum depression scores.
\end{abstract}

Methods: This study used data from the COVID-19 And Reproductive Effects (CARE) study, an online survey of pregnant persons in the United States. Participants completed postnatal surveys between April 30 - November 18, 2020 $(n=971)$. Logistic regression analysis tested whether birth timing during the pandemic was associated with odds of reported sustained instrumental support. Linear regression analyses assessed whether instrumental support was associated with lower depression scores as measured via the Edinburgh Postnatal Depression survey.

Results: Participants who gave birth later in the pandemic were more likely to report that the pandemic had not affected the help they received with household work and newborn care $(p<0.001)$, while access to childcare for older children appeared to vary non-linearly throughout the pandemic. Additionally, respondents who reported that the pandemic had not impacted their childcare access or help received around the house displayed significantly lower depression scores compared to participants who reported pandemic-related disruptions to these support types $(p<0.05)$.

Conclusions: The maintenance of postpartum instrumental support during the pandemic appears to be associated with better maternal mental health. Healthcare providers should therefore consider disrupted support systems as a risk factor for postpartum depression and ask patients how the pandemic has affected support access. Policymakers seeking to improve parental wellbeing should design strategies that reduce disease transmission, while facilitating safe interactions within immediate social networks (e.g., through investment in COVID-19 testing and contact tracing).

\footnotetext{
*Correspondence: gildner@wustl.edu

${ }^{1}$ Department of Anthropology, Washington University in St. Louis, St.

Louis, MO, USA

Full list of author information is available at the end of the article
} original author(s) and the source, provide a link to the Creative Commons licence, and indicate if changes were made. The images or other third party material in this article are included in the article's Creative Commons licence, unless indicated otherwise in a credit line to the material. If material is not included in the article's Creative Commons licence and your intended use is not permitted by statutory regulation or exceeds the permitted use, you will need to obtain permission directly from the copyright holder. To view a copy of this licence, visit http://creativecommons.org/licenses/by/4.0/. The Creative Commons Public Domain Dedication waiver (http://creativeco mmons.org/publicdomain/zero/1.0/) applies to the data made available in this article, unless otherwise stated in a credit line to the data. 
Cumulatively, postpartum instrumental support represents a potential tool to protect against depression, both during and after the COVID-19 pandemic.

Keywords: Mental health, Perinatal depression, Maternal wellbeing, Coronavirus, Social support, Childcare

\section{Background}

The early postpartum period represents a time of recovery and adjustment. In many cultures, the first 30-45 days are characterized by a set of common rituals, including rest periods and prescribed dietary and hygiene regimes believed to support maternal physical and mental health $[1,2]$. Within the United States, however, less attention has been paid to postpartum health beyond the first few days following delivery [1]. Moreover, fewer formal mechanisms designed to support postpartum wellbeing, including federally mandated paid parental leave, exist within the U.S., potentially undermining maternal recuperation by exacerbating physical fatigue and compromising mental health ( $[1,2])$. Postpartum health and mental wellbeing (including depression risk) are influenced by many biocultural factors, including experienced racism and racial disparities in resource access, pregnancy intendedness (i.e., whether the pregnancy was planned), previous experiences of trauma or poor physical and mental health, work-related anxiety, financial stress, and physical activity patterns [3-5]. Social support from partners, family members, and community members represents another important factor impacting postpartum recovery and health [3, 6-9], and some level of postpartum support is common in many American communities. New parents commonly receive support from family, friends, and neighbors as they adjust to life with a newborn. This support comes in different forms, including emotional and informational support [10-13]. However, another important type of postpartum assistance is instrumental support, which includes activities such as help with childcare and other household tasks (e.g., cleaning and housework preparation) [10-13].

Extended family members are generally an important source of instrumental support, often assisting with newborn care and daily household tasks during the postpartum period, allowing mothers to have needed time to themselves [14-16]. Instrumental support is therefore essential for supporting maternal wellbeing following childbirth, with evidence demonstrating that women consider this type of support from friends and family a critical part of their physical and emotional recovery $[14,15]$. Conversely, mothers who receive less postpartum instrumental support exhibit an elevated risk for postpartum depression (PPD); for example, individuals in one study who reported receiving low levels of instrumental support were approximately five times more likely to develop PPD as mothers who reported high levels of instrumental support [17].

The positive impact of instrumental support on maternal wellbeing is not trivial, as new mothers may be at an especially high risk of developing depressive symptoms. The perinatal period is associated with a range of physical and physiological changes, often leading to increased levels of stress, fear, and anxiety with the transition to parenthood [8]. Postpartum individuals consequently exhibit elevated depression rates compared to the general public, such that as many as 1 in 8 women in the U.S. have been estimated to experience PPD symptoms ( [18, 19]). Postpartum depression can have serious effects not only on maternal quality of life (e.g., resulting in poor sleep quality, loss of appetite, lasting sadness, anxiety, thoughts and/or attempts to harm oneself or the baby), but may also negatively affect the infant. Maternal PPD can lead to difficulty in breastfeeding, poor maternal and infant bonding, and delays in multiple aspects of infant development which may increase the risk of early noncommunicable disease onset during adulthood [20]. Thus, reducing PPD risk is important for both maternal and infant wellbeing, and enhanced support during the postpartum period may represent one important nonpharmaceutical strategy for supporting maternal mental health. Instrumental support in particular may protect against PPD, as this form of support has been shown to reduce the care burdens placed on mothers while also signaling that they are loved and valued ( $[16,21])$.

Yet, the COVID-19 pandemic has disrupted daily life with implications for access to common forms of instrumental support postpartum. Travel restrictions tied with stay-at-home orders may prevent family and friends from visiting for the birth and early postpartum period [22], potentially decreasing the amount of help received with household tasks and newborn care. In addition, other sources of support may be disrupted, including access to school and daycare for older children [23, 24]. These school and childcare closures appear to especially impact mothers, who have disproportionately provided childcare and supervised remote learning during the COVID-19 pandemic [23, 24]. Cumulatively, these instrumental support disruptions may increase PPD risk among mothers living in the U.S., although this remains to be directly tested. Still, evidence in other countries suggests that PPD rates have risen during the pandemic, and that disrupted instrumental support may partly account for 
this pattern. For example, studies conducted in China and Italy indicate a high prevalence of PPD among individuals during the COVID-19 pandemic (30 and 44\%, respectively), while reduced contact with loved ones was significantly associated with PPD $[25,26]$. Further work is needed, however, to determine whether specific aspects of postpartum instrumental support may protect against PPD during the ongoing pandemic.

The impact of the COVID-19 pandemic on instrumental support access has likely varied over time, as rates of disease transmission and associated social distancing and travel recommendations fluctuate. Evidence indicates that stay-at-home orders imposed by local governments have changed over time, with widespread lockdowns at the start of the pandemic and a gradual easing of restrictions in subsequent months $[27,28]$. Reduced mobility in the U.S. is clearly correlated with shelter-in-place restrictions, such that areas with official orders (more common in April than during the June-August) exhibited substantially greater reductions in movement compared to locations without stay-at-home orders [28, 29]. It therefore seems likely that pandemic-related changes to postpartum instrumental support have not remained constant, although this has not yet been fully explored. To address these issues, we use data from the COVID19 and Reproductive Effects (CARE) study - an online survey of pregnant and postpartum persons living in the U.S. which assesses how the COVID-19 pandemic has affected maternal wellbeing. The aims of this study were to evaluate:

1) Whether the level of instrumental support (in the form of assistance with housework and newborn care or childcare for older children) reported within the first few weeks of the postpartum period has changed across the pandemic (April - November 2020).

2) Whether reported instrumental support during the COVID-19 pandemic is associated with lower maternal depression scores, as measured by the Edinburgh postnatal depression survey.

\section{Methods Study design}

The COVID-19 And Reproductive Effects (CARE) study was posted on social media platforms (Facebook, Twitter) and distributed via email to contacts working in maternity care and public health. Pregnant persons over the age of 18 and living in the United States were invited to participate in a short survey assessing how the COVID-19 pandemic had impacted their healthcare and wellbeing. Participants who agreed to be recontacted received a postnatal survey four weeks after their due date. The postnatal data presented here were collected between April 30 - November 18, 2020. This study received ethical approval from Dartmouth College (STUDY00032045) and all research was performed in accordance with the Declaration of Helsinki. Informed consent was obtained from all participants. The survey was administered using REDCap (Research Electronic Data Capture) hosted through Dartmouth College. REDCap is a secure web platform that facilitates the creation and management of online surveys for research studies $[30,31]$. The survey completion rate (i.e., the percentage of those who consented to take the survey and actually went through to the end of the questionnaire) was $92.8 \%$ (1033/1113 participants). During the study period, there were 976 surveys collected that included responses for all study variables. Data on depression symptomatology and support systems were collected, along with other covariates known to influence depression risk.

Depression scores Depression symptoms were screened using the gold-standard Edinburgh Postnatal Depression Survey (EPDS). The EPDS is a self-report 10-question instrument based on individual experiences in the previous seven days. This well validated scale is designed to measure various aspects of clinical depression, including reports of feeling guilty, disrupted sleep, low energy, inability to feel pleasure, and suicidal ideation. The responses are scored and summed, resulting in a participant score ranging from 0 (minimum, little indication of depressive symptoms) to 30 (maximum, high likelihood of depression) [32].

Postpartum social support Participants were asked whether the COVID-19 pandemic had led to them receiving less help and support with household tasks and newborn care (yes/no).

Childcare support Respondents were asked, "If you have other children, has/did the COVID-19 pandemic affect your access to childcare?" These data were analyzed for the subset of participants in the dataset with other children $(n=398)$ to determine whether childcare disruptions varied over the course pandemic or were related to maternal PPD. Specifically, individuals who responded that their other child(ren)'s daycare had closed (either temporarily or permanently) or that their other child(ren) could no longer be cared for by others (e.g., a nanny or a relative) were coded as experiencing disrupted childcare. Conversely, participants who indicated that their other child(ren) were never cared for by others outside of their household or that their other child(ren) continued to go to daycare were coded as experiencing sustained childcare. 
Birth date Participants reported when they gave birth. This date was then used to calculate how far into the pandemic the participant gave birth. Specifically, the number of days between March 11, 2020 (the day the WHO officially declared COVID-19 a pandemic) and the day of birth was calculated, such that larger values reflect giving birth later in the course of the pandemic.

Postpartum duration Time into the postpartum period may influence access to support systems (e.g., individuals may experience increased levels of support immediately after giving birth as compared to three months later). Additionally, postpartum period length also appears to influence the risk of developing depression [33, 34]. It is therefore important to account for postpartum duration when considering access to instrumental support and depression symptoms during the postnatal period. The number of days that had passed between giving birth and completing the postpartum survey was consequently calculated for each participant.

Maternal age Past research indicates that maternal age is inversely related to depressive symptoms [35]. Thus, participants self-reported their age in years.

Race/ethnicity Race/ethnicity has been linked with maternal depression risk, with minority populations exhibiting higher depression rates [35]. Participant race/ ethnicity was therefore self-reported and measured according to the Office of Management and Budget Standards [36]. Native Hawaiian/Pacific Islander participants were re-classified as "Other" due to a small sample size $(n=3)$. This categorical variable was dummy coded during analysis, with "white" serving as the reference group.

Household income Previous work indicates that higher income levels may protect against maternal depression [35]. Participants were thus asked to select their household income from the following options: Less than $\$ 10,000$ (1); \$10,000 - \$19,999 (2); \$20,000 - \$34,999 (3); $\$ 35,000$ - \$49,999 (4); $\$ 50,000$ - \$74,999 (5); $\$ 75,000$ - \$99,999 (6); $\$ 100,000+(7)$. A composite household income variable was created for analysis: < \$49,999, $\$ 50,000-\$ 99,999$, and $\$ 100,000+(<\$ 49,999$ serves as the reference group in analysis).

Education Lower education levels have been linked with increased depression risk during pregnancy [35]. Participants consequently selected their highest completed education from the following options: Some high school, no diploma (1); High school graduate, diploma or the equivalent (for example: GED) (2); Some college credit, no degree (3); Trade/technical/vocational training (4); Associate degree (5); Bachelor's degree (6); Master's degree (7); Professional degree (8); Doctorate degree (9). A composite education variable was created for analysis: less than a bachelor's degree, a bachelor's degree, or a degree beyond a bachelor's degree (less than a bachelor's degree serves as the reference group in analysis).

\section{Statistical analysis}

Data analyses were conducted using Stata 14. All continuous variables exhibited normal distributions, with skewness values within \pm 1 . Multicollinearity was not detected between any variables; all VIF values were in an acceptable range of 1.02-1.44. A plot of the linear regression model residuals versus fitted values did not indicate that heteroscedasticity was a concern. Five outliers were identified. Specifically, five participants exhibited extreme duration values between giving birth and completing the survey. Two respondents apparently completed the postpartum survey 13 and 2 days before giving birth, while another three waited months to complete the survey (i.e., 113, 114 and 129 days passed between giving birth and completing the survey). These five participants were consequently excluded from the analyses so that the analyses only accounted for depression scores and social support experiences within the first few months of giving birth (range 2-89 days following delivery). This resulted in a final sample size of 971 participants; a power analysis (for a linear multiple regression model with an estimated 6 predictors, power 0.80 , and alpha 0.04 ) indicated that this sample size would have the sensitivity to detect an effect size $\mathrm{f}^{2}$ of 0.006 , a very small effect. Study descriptive statistics were calculated, and regression analyses were conducted to test the study hypotheses. Results were considered statistically significant at $p<0.05$.

Logistic regression analyses assessed whether later birth date (signifying later in the course of the pandemic) was significantly associated with an increased likelihood of reporting help with household work/newborn care due to the easing of initial lockdown measures. In other words, it was hypothesized that individuals giving birth later during the pandemic may have faced less severe shelter-in-place restrictions, and consequently would have been more likely to report that the pandemic had not reduced help received around the house. Conversely, the question about care for older children asked about care disruptions at any point during the pandemic. It was therefore hypothesized that later birth date would be associated with higher odds of reporting childcare disruptions (i.e., since more time had passed during which care may have been affected).

In addition, linear regression analyses were used to assess whether participants who reported instrumental support exhibited significantly lower depression scores. 
These analyses were run with the full sample and a second time excluding participants who completed the study survey within the first two weeks of giving birth $(N=51$ participants), to account for the possibility that the EPDS score was capturing "baby blues" (i.e., shortlasting depressive-like symptoms that may immediately follow delivery) instead of PPD [37]. However, the results did not qualitatively differ between the two models. The full sample was therefore retained to enhance statistical power. All analyses adjusted for maternal age, education, household income, race/ethnicity, postpartum duration, and time between pandemic onset.

\section{Results}

Sample characteristics and descriptive statistics

Sample descriptive statistics are presented in Table 1. Mean participant age was 32 years old. Most respondents were white $(90 \%$ of the sample), educated ( $85 \%$ had at least a bachelor's degree) and had higher income (61\% reported an annual household income of $\$ 100,000$ or more). The majority of participants reported that they were receiving less help and support with household work and/or newborn care $(60 \%)$ that they attributed to the COVID-19 pandemic; likewise, among the subset of respondents who had other children, many reported their childcare access had been disrupted (57\%). Finally, participants varied in depression scores as measured by the EPDS scale, ranging from the minimum score of 0 to 24; the mean EPDS score was 7 and approximately $11 \%$ of the sample displayed clinically significant EPDS scores indicative of probable major depression (using a conservative cutoff of $\geq 13$ ) [38].

\section{Postpartum instrumental support in relation to date of giving birth}

Logistic regression analyses were carried out to determine whether giving birth later in the course of the

Table 1 Descriptive statistics of model variables

\begin{tabular}{|c|c|}
\hline Variable & Mean (SD; range) \\
\hline Age (years) & $31.9(4.0 ; 18-47)$ \\
\hline Edinburgh Postnatal Depression Survey (EPDS) score & $6.96(4.2 ; 0-24)$ \\
\hline Number of days between onset of pandemic and giving birth & $118(49.9 ; 13-235)$ \\
\hline \multirow[t]{2}{*}{ Number of days between giving birth and completing the study survey } & $30.7(12.4 ; 2-89)$ \\
\hline & Frequency (\%) \\
\hline \multicolumn{2}{|l|}{ Clinically significant EPDS scores } \\
\hline$<13$ (clinical depression unlikely) & $868(89.4 \%)$ \\
\hline$\geq 13$ (probable clinical depression) & $103(10.6 \%)$ \\
\hline \multicolumn{2}{|l|}{ Race/ethnicity: } \\
\hline White & $872(89.8 \%)$ \\
\hline Hispanic, Latino, or Spanish origin & $42(4.3 \%)$ \\
\hline Black or African American & $10(1.0 \%)$ \\
\hline Asian & $25(2.6 \%)$ \\
\hline American Indian or Alaskan Native & $5(0.5 \%)$ \\
\hline Other & $17(1.8 \%)$ \\
\hline \multicolumn{2}{|l|}{ Household income: } \\
\hline$<\$ 49,999$ & $87(9.0 \%)$ \\
\hline$\$ 50,000-\$ 99,999$ & $292(30.1 \%)$ \\
\hline$\$ 100,000+$ & $592(61.0 \%)$ \\
\hline \multicolumn{2}{|l|}{ Education level: } \\
\hline Less than a bachelor's degree & $147(15.1 \%)$ \\
\hline Bachelor's degree & $342(35.2 \%)$ \\
\hline Degree beyond a bachelor's degree & $482(49.7 \%)$ \\
\hline \multicolumn{2}{|l|}{ Less support in housework/newborn care due to pandemic } \\
\hline Yes & $582(59.9 \%)$ \\
\hline No & $389(40.1 \%)$ \\
\hline \multicolumn{2}{|c|}{ Childcare access during pandemic (among subset of participants with other children, $n=395$ ) } \\
\hline Affected & $227(57.5 \%)$ \\
\hline Unaffected & $168(42.5 \%)$ \\
\hline
\end{tabular}

Sample means (with standard deviation and range) or frequency (percent) of model variables, for 971 participants included in the analyses 
pandemic was significantly associated with an increased likelihood of reporting sustained support with household work and newborn care or childcare access (Tables 2 and 3). Preliminary regression analyses assessed whether the likelihood of reporting support varied non-linearly with birth date. No significant non-linear associations were observed in the model assessing the relationship between birth timing and support with household work and newborn care; however, a significant cubic trend was evident between timing of birth in the course of the pandemic and childcare access among the subset of women who had other children $(p=0.048)$.

In the model assessing the association between time of birth and likelihood of reporting continued help around the house and with newborn care, participants who were older $(\mathrm{OR}=0.916,95 \% \mathrm{CI}$ : $0.882-0.951, p<0.001)$ and who were more highly educated (reference: less than a bachelor's degree; bachelor's degree $\mathrm{OR}=0.563$, 95\%CI: $0.367-0.864, p=0.009$; degree beyond a bachelor's degree $\mathrm{OR}=0.585,95 \% \mathrm{CI}$ : $0.379-0.904, p=0.016$ )

Table 2 Logistic regression model assessing the association between timing of birth during the pandemic and the likelihood of reporting sustained help with household tasks and newborn care

\begin{tabular}{|c|c|c|}
\hline Variable & OR (SE, 95\% Cl) & $p$-value \\
\hline Intercept & $6.31(3.87,1.90-21.0)$ & 0.003 \\
\hline Age (years) & $0.916(0.018,0.882-0.951)$ & $<0.001$ \\
\hline \multicolumn{3}{|l|}{ Race/ethnicity: } \\
\hline White & Reference & \\
\hline $\begin{array}{l}\text { Hispanic, Latino, or Spanish } \\
\text { origin }\end{array}$ & $0.588(0.211,0.291-1.19)$ & 0.140 \\
\hline Black or African American & $5.54(3.98,1.36-22.6)$ & 0.017 \\
\hline Asian & $1.98(0.823,0.873-4.47)$ & 0.102 \\
\hline $\begin{array}{l}\text { American Indian or Alaskan } \\
\text { Native }\end{array}$ & $0.627(0.615,0.092-4.29)$ & 0.635 \\
\hline Other & $2.43(1.23,0.901-6.57)$ & 0.079 \\
\hline \multicolumn{3}{|l|}{ Household income: } \\
\hline$<\$ 49,999$ & reference & \\
\hline$\$ 50,000-\$ 99,999$ & $1.51(0.407,0.893-2.56)$ & 0.124 \\
\hline$\$ 100,000+$ & $1.33(0.364,0.778-2.28)$ & 0.297 \\
\hline \multicolumn{3}{|l|}{ Education level: } \\
\hline Less than a bachelor's degree & reference & \\
\hline Bachelor's degree & $0.563(0.123,0.367-0.864)$ & 0.009 \\
\hline $\begin{array}{l}\text { Degree beyond a bachelor's } \\
\text { degree }\end{array}$ & $0.585(0.130,0.379-0.904)$ & 0.016 \\
\hline $\begin{array}{l}\text { Number of days between giving } \\
\text { birth and completing the study } \\
\text { survey }\end{array}$ & $1.00(0.006,0.990-1.01)$ & 0.789 \\
\hline $\begin{array}{l}\text { Number of days between the } \\
\text { onset of the pandemic and giv- } \\
\text { ing birth }\end{array}$ & $1.01(0.001,1.00-1.01)$ & $<0.001$ \\
\hline
\end{tabular}

Odds ratios are provided with standard errors, $95 \%$ confidence intervals, and $p$-values for each variable included in the model
Table 3 Logistic regression model assessing the association between timing of birth during the pandemic and the likelihood of reporting continued childcare access, from a subset of participants with other children $(n=393)$. Odds ratios are provided with standard errors, 95\% confidence intervals, and $p$-values for each variable included in the model

\begin{tabular}{|c|c|c|}
\hline Variable & OR (SE, 95\% Cl) & $p$-value \\
\hline Intercept & $0.310(0.958,0.001-133)$ & 0.705 \\
\hline Age (years) & $0.956(0.032,0.897-1.02)$ & 0.183 \\
\hline \multicolumn{3}{|l|}{ Race/ethnicity: } \\
\hline White & reference & \\
\hline $\begin{array}{l}\text { Hispanic, Latino, or Spanish } \\
\text { origin }\end{array}$ & $0.899(0.508,0.297-2.72)$ & 0.851 \\
\hline Black or African American & $1.57(1.54,0.231-10.8)$ & 0.643 \\
\hline Asian & $1.14(1.18,0.152-8.63)$ & 0.896 \\
\hline Other & $1.46(0.333,0.366-1.81)$ & 0.616 \\
\hline \multicolumn{3}{|l|}{ Household income: } \\
\hline$<\$ 49,999$ & reference & \\
\hline$\$ 50,000-\$ 99,999$ & $1.65(0.661,0.758-3.62)$ & 0.206 \\
\hline$\$ 100,000+$ & $0.815(0.333,0.366-1.81)$ & 0.616 \\
\hline \multicolumn{3}{|l|}{ Education level: } \\
\hline Less than a bachelor's degree & reference & \\
\hline Bachelor's degree & $0.588(0.205,0.298-1.16)$ & 0.128 \\
\hline $\begin{array}{l}\text { Degree beyond a bachelor's } \\
\text { degree }\end{array}$ & $0.270(0.100,0.130-0.560)$ & $<0.001$ \\
\hline $\begin{array}{l}\text { Number of days between giving } \\
\text { birth and completing the study } \\
\text { survey }\end{array}$ & $0.994(0.010,0.974-1.01)$ & 0.581 \\
\hline \multicolumn{3}{|l|}{$\begin{array}{l}\text { Number of days between the } \\
\text { onset of the pandemic and giv- } \\
\text { ing birth: }\end{array}$} \\
\hline Time between & $1.10(0.072,0.963-1.25)$ & 0.164 \\
\hline Time between squared & $0.999(0.001,0.998-1.00)$ & 0.094 \\
\hline Time between cubed & $1.00(1.22 \mathrm{e}-6,1.00-1.00)$ & 0.048 \\
\hline
\end{tabular}

were significantly less likely to report sustained instrumental support related to household tasks and newborn care during the pandemic. Conversely, compared to white participants, Black or African American participants were more likely to report that the pandemic had not impacted their support with household tasks and newborn care $(\mathrm{OR}=5.54,95 \% \mathrm{CI}: 1.36-22.61, p=0.017)$. Finally, as hypothesized, participants who gave birth later in the course of the pandemic were more likely to report that the COVID-19 pandemic had not affected help received with household tasks and newborn care $(\mathrm{OR}=1.01,95 \% \mathrm{CI}: 1.00-1.01, p<0.001)$ (Table 2). Timing of birth during the pandemic was calculated in days to better capture rapidly shifting shelter-in-place recommendations that may have impacted instrumental support access, while also allowing us to more closely examine the non-linear association between birth timing and childcare access (see below). However, the significant 
association between birth timing and sustained household help documented here was consistent when timing of birth was calculated in weeks $(\mathrm{OR}=1.04,95 \% \mathrm{CI}$ : 1.02 $1.06, p<0.001)$ or months (OR $=1.17,95 \% \mathrm{CI}: 1.08-1.27$, $\mathrm{p}<0.001$.

Among the subset of participants with other children, mothers who were very highly educated were more likely to report disrupted childcare access (reference: less than a bachelor's degree; degree beyond a bachelor's: $\mathrm{OR}=0.270,95 \% \mathrm{CI}$ : $0.130-0.560, \mathrm{p}<0.001$ ). Further, the addition of a cubic term was significant $(\mathrm{B}=1.00,95 \% \mathrm{CI}: 1.00-1.00001, \mathrm{p}=0.048)$, suggesting a non-linear relationship in childcare access across the course of the pandemic (Table 3). Specifically, the fitted cubic trendline suggested that childcare access increased substantially toward the end of August ( day 170 of the pandemic).

To explore this seemingly paradoxical relationship (i.e., that a greater number of participants reported that the pandemic had not affected their childcare later in the course of the pandemic when more time had passed during which childcare may have been disrupted), moving averages over the previous 30 days were graphed to determine whether response rates for either specific "care unaffected" response were driving this pattern (Fig. 1). Moving (or rolling) averages are derived from successive means over periods of a defined length (e.g., a number of days) and are commonly used to visualize trends over time [39]. A moving average graph of these data suggests that, among individuals reporting sustained access to childcare, the proportion of participants reporting their child(ren) had never been cared for outside of the household generally decreased throughout the pandemic, while the proportion of participants reporting their child(ren) continued to go to daycare simultaneously increased (especially after late August, driving the observed unusual pattern).

The averages reflect the proportion of participants reporting sustained childcare access because child(ren) never cared for by anyone outside of the house (in red) compared to the proportion of participants reporting sustained childcare access because child(ren) continued to go to daycare (in blue) in relation to days between the start of the pandemic and giving birth.

\section{Postpartum support and maternal depression}

Linear regression analyses were carried out to assess whether early postpartum instrumental support was significantly associated with depression score, measured using the EPDS (Tables 4 and 5). Participants who were older $(\mathrm{B}=-0.101,95 \% \mathrm{CI}:-0.172-(-0.031), p=0.005)$, reported a higher household income (reference: < $\$ 49,999 ; \quad \$ 50,000-\$ 99,999 \mathrm{~B}=-1.20,95 \% \mathrm{CI}:-2.22$ $(-0.180), \quad p=0.021 ; \quad \$ 100,000+\quad \mathrm{B}=-1.27, \quad 95 \% \mathrm{CI}$ : $-2.31-(-0.236), p=0.016)$, and were more highly educated (reference: less than a bachelor's degree; bachelor's degree $\mathrm{B}=-1.06,95 \% \mathrm{CI}:-1.90-(-0.212), p=0.014$; degree beyond a bachelor's degree $\mathrm{B}=-1.02$, 95\%CI: $-1.87-(-0.159), p=0.020)$ exhibited significantly lower depression scores. Participants further into their postpartum period (i.e., who exhibited a greater duration of time between giving birth and completing the survey) displayed significantly higher depression scores $(\mathrm{B}=0.023$, 95\%CI: 0.002-0.045, $p=0.033)$. As expected,

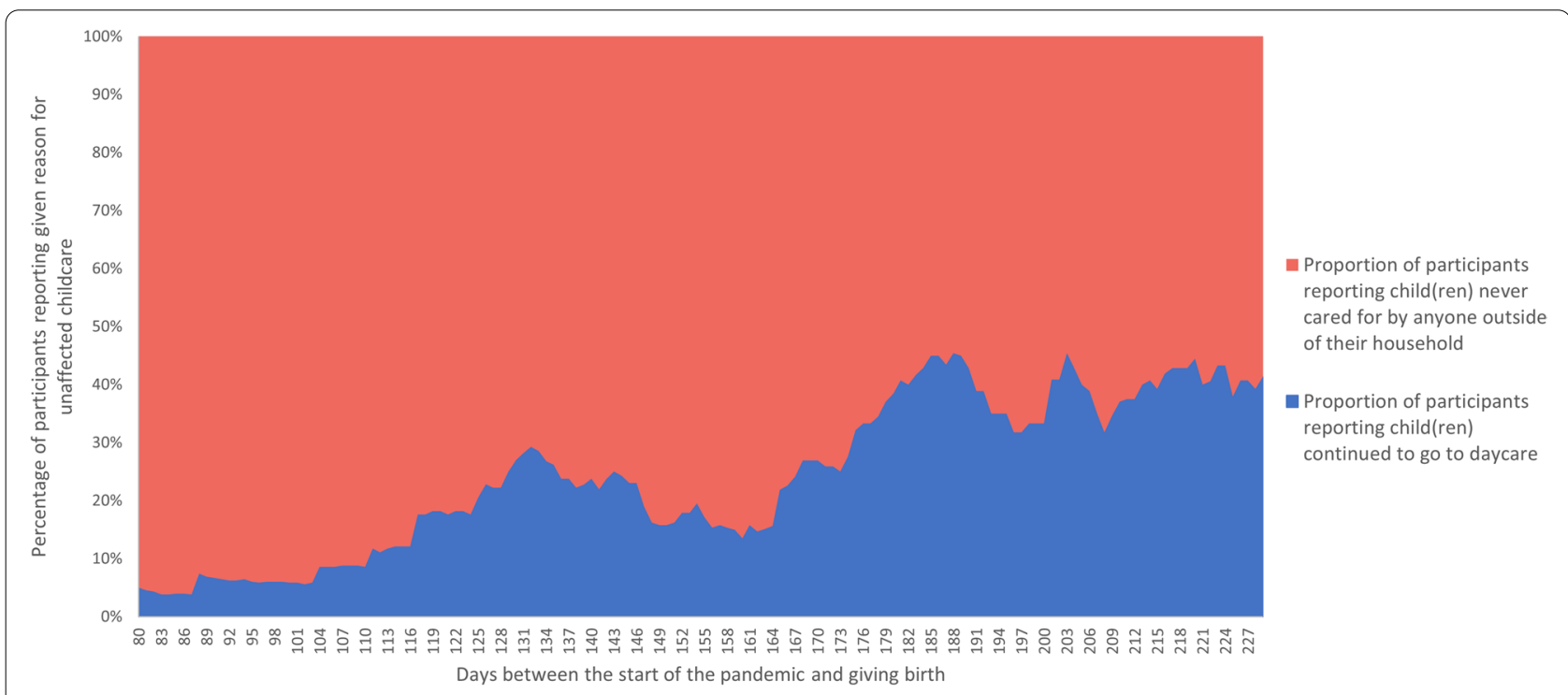

Fig. 1 Reason for reporting childcare access was unaffected, plotted as a 30-day moving average 
Table 4 Linear regression model assessing the association between reported help with household tasks/newborn care during the COVID-19 pandemic and Edinburgh Postnatal Depression Survey (EPDS) score

\begin{tabular}{|c|c|c|}
\hline Variable & B coefficient ( $\mathrm{SE}, 95 \% \mathrm{Cl})$ & $p$-value \\
\hline Intercept & $12.2(1.19,9.85-14.5)$ & $<0.001$ \\
\hline Age (years) & $-0.101(0.036,-0.172-(-0.031))$ & 0.005 \\
\hline \multicolumn{3}{|l|}{ Race/ethnicity: } \\
\hline White & reference & \\
\hline Hispanic, Latino, or Spanish origin & $0.981(0.649,-0.292-2.26)$ & 0.131 \\
\hline Black or African American & $2.25(1.31,-0.319-4.82)$ & 0.086 \\
\hline Asian & $-0.513(0.828,-2.14-1.11)$ & 0.536 \\
\hline American Indian or Alaskan Native & $3.15(1.83,-0.445-6.74)$ & 0.086 \\
\hline Other & $0.662(0.998,-1.30-2.62)$ & 0.507 \\
\hline \multicolumn{3}{|l|}{ Household income: } \\
\hline$<\$ 49,999$ & reference & \\
\hline$\$ 50,000-\$ 99,999$ & $-1.20(0.520,-2.22-(-0.180))$ & 0.021 \\
\hline$\$ 100,000+$ & $-1.27(0.528,-2.31-(-0.236))$ & 0.016 \\
\hline \multicolumn{3}{|l|}{ Education level: } \\
\hline Less than a bachelor's degree & reference & \\
\hline Bachelor's degree & $-1.06(0.430,-1.90-(-0.212))$ & 0.014 \\
\hline Degree beyond a bachelor's degree & $-1.02(0.436,-1.87-(-0.159))$ & 0.020 \\
\hline Number of days between giving birth and completing the study survey & $0.023(0.011,0.002-0.045)$ & 0.033 \\
\hline Number of days between the onset of the pandemic and giving birth & $-0.002(0.003,-0.007-0.003)$ & 0.410 \\
\hline Help with household tasks and newborn care affected by the pandemic (yes vs no) & $-1.27(0.275,-1.81-(-0.731))$ & $<0.001$ \\
\hline
\end{tabular}

Beta coefficients are provided with standard errors, $95 \%$ confidence intervals, and $p$-values for each variable included in the model

Table 5 Linear regression model assessing the association between reported childcare disruptions during the COVID-19 pandemic and Edinburgh Postnatal Depression Survey (EPDS) score, from a subset of participants with other children ( $n=395)$. Beta coefficients are provided with standard errors, 95\% confidence intervals, and $p$-values for each variable included in the model

\begin{tabular}{|c|c|c|}
\hline Variable & B coefficient ( $\mathrm{SE}, 95 \% \mathrm{Cl})$ & p-value \\
\hline Intercept & $9.20(1.99,5.30-13.1)$ & $<0.001$ \\
\hline Age (years) & $-0.085(0.058,-1.99-0.029)$ & 0.143 \\
\hline \multicolumn{3}{|l|}{ Race/ethnicity: } \\
\hline White & reference & \\
\hline Hispanic, Latino, or Spanish origin & $0.663(0.999,-1.30-2.63)$ & 0.507 \\
\hline Black or African American & $1.06(1.76,-2.40-4.51)$ & 0.548 \\
\hline Asian & $0.708(1.96,-3.15-4.57)$ & 0.718 \\
\hline American Indian or Alaskan Native & $4.93(2.80,-0.574-10.4)$ & 0.079 \\
\hline Other & $-0.665(1.40,-3.42-2.09)$ & 0.635 \\
\hline \multicolumn{3}{|l|}{ Household income: } \\
\hline$<\$ 49,999$ & reference & \\
\hline$\$ 50,000-\$ 99,999$ & $-0.859(0.725,-2.28-0.566)$ & 0.237 \\
\hline$\$ 100,000+$ & $-0.937(0.745,-2.40-0.527)$ & 0.209 \\
\hline \multicolumn{3}{|l|}{ Education level: } \\
\hline Less than a bachelor's degree & reference & \\
\hline Bachelor's degree & $-0.548(0.627,-1.78-0.685)$ & 0.383 \\
\hline Degree beyond a bachelor's degree & $-0.799(0.674,-2.12-0.526)$ & 0.237 \\
\hline Number of days between giving birth and completing the study survey & $0.056(0.017,0.023-0.089)$ & 0.001 \\
\hline Number of days between the onset of the pandemic and giving birth & $0.001(0.005,-0.008-0.010)$ & 0.872 \\
\hline Childcare access (affected vs. unaffected) & $-1.03(0.421,-1.86-(-0.201))$ & 0.015 \\
\hline
\end{tabular}


participants who reported that the pandemic had not disrupted access to help and support with household tasks and newborn care during the pandemic displayed significantly lower depression scores than participants who reported they had received less support due to the pandemic $(\mathrm{B}=-1.27,95 \% \mathrm{CI}:-1.81-(-0.731), p<0.001$, $\left.\eta^{2}=0.02\right)$ (Table 4).

An additional regression analysis was performed to assess the relationship between depression score and childcare access among the subset of mothers with older children. Participants further into their postpartum period exhibited significantly higher depression scores $(\mathrm{B}=0.056,95 \% \mathrm{CI}$ : 0.023-0.089, $p=0.001)$. As hypothesized, participants who reported their childcare had not been disrupted exhibited significantly lower depression scores compared to individuals who reported their childcare had been affected by the pandemic $(\mathrm{B}=-1.03$, 95\% CI: $-1.86-(-0.201), p=0.015$, $\left.\eta^{2}=0.02\right)$ (Table 5).

\section{Discussion}

The study findings provide support for both hypotheses. Participants who gave birth later in the course of the pandemic (range April to November 2020) were more likely to report that the pandemic had not affected the support they received with household work and newborn care. This suggests that individuals who gave birth earlier in the pandemic, when lockdowns were more prevalent and general mobility was lower, were more likely to experience disruptions to some aspects of instrumental support. The results also suggest that maintenance of instrumental support during the pandemic may have played an important role in supporting maternal mental health, although it should be noted the effect sizes were rather small. Still, the relationships between the instrumental support measures and PPD score were statistically significant. Specifically, mothers who reported that the pandemic had not negatively impacted their access to childcare or the help they received with housework and newborn care displayed significantly lower depression scores compared to participants who reported pandemic-related disruptions in access to instrumental support. One important strength of the present study is that it focused on protective factors that may improve postpartum wellbeing. Many studies focus on risk factors associated with elevated depression risk $[3,19,40,41]$, including studies examining poor mental health during the COVID-19 pandemic $[42,43]$. While this research is important, it is also necessary to frame data analyses and interpretation in a more positive light, including efforts to highlight factors that appear to enhance (rather than reduce) postpartum health.

\section{Changes in access to instrumental support throughout the course of the pandemic}

The results presented here suggest that perceived support related to household work and newborn care has varied throughout the course of the pandemic in the U.S., with participants giving birth later in the pandemic being more likely to report that the pandemic had not affected the social support they received around the house and with newborn care. This pattern may be evident for a couple of reasons. First, participants giving birth later into the pandemic may have benefited from relaxed shelter-in-place restrictions that had by then been instituted in many areas of the country. Mobility data collected using location data stored on Google and Apple devices indicate that mobility in the U.S. was greatly curtailed at the start of the pandemic $[28,29]$, but that mobility generally increased in the following months as infection spread was reduced or government officials were pressured to ease restrictions as pandemic fatigue increased and citizens were less willing to follow stay-at-home orders [44].

Thus, as shelter-in-place restrictions eased after the first few months of the pandemic in many areas, mothers giving birth during this time may have felt safer receiving support from friends and family around the house during the newborn period. However, this remains to be explicitly tested. Future studies should also test whether individuals in the early postpartum period during the nationwide surge of COVID-19 cases at the end of 2020 and start of 2021 (and associated rise in state restrictions aimed at controlling disease transmission) also report restricted access to childcare and less support around the house, as was documented in the early days of the pandemic when stay-at-home orders were more widespread. In addition to benefiting from relaxed shelter-in-place restrictions, participants who gave birth months into the course of the pandemic in our sample may have also had more time to adjust to the new reality of the pandemic and develop alternative support networks. For example, individuals may have moved in with family members to shelter-in-place together, or they may have formed pandemic "pods" with other families in the area, an idea that has received attention in the media $[45,46]$. These new networks may have increased the amount of instrumental support received during the postpartum period. Future work should assess how individuals have shown resilience and shifted their support systems in response to the ongoing pandemic.

However, access to childcare appeared to vary nonlinearly throughout the course of the pandemic, such that higher rates of participants perplexingly reported continual access to childcare later during the pandemic. A graph of moving 30-day averages indicates that a higher 
percentage of participants with unaffected care reported continued access to daycare later in the pandemic (compared to the percentage reporting that their children had never been cared for by anyone outside the household). It does appear, however, that the rise in respondents indicating that daycare remained unaffected rose noticeably starting at the end of August 2020. It therefore seems likely that this rise could coincide with the start of the school year. School attendance was not explicitly listed as one of the childcare options; it is consequently possible that participants who gave birth later in the course of the pandemic selected the response "my other child(ren) continued to go to daycare" to include children enrolled in school, while respondents earlier in the pandemic were less likely to respond in this manner because older children were out of school due to initial lockdowns or because of summer vacation when American schools are not in session. Additional data collection is needed to explore whether school openings at the start of the academic year were directly related to reports of.

\section{The importance of instrumental support in supporting maternal mental health}

The results of the present study align well with previous work documenting the importance of received support in protecting maternal mental health during the postpartum period. Notably, received support was significantly associated with PPD in this relatively privileged sample (i.e., participants were predominantly white, wealthy, and highly educated); but other work has found social support is especially important in protecting mental health among vulnerable groups (e.g., minoritized communities, individuals with a history of trauma and poor mental health, and those in unstable living conditions or with unreliable healthcare access) [3, 8, 9, 47, 48]. Maternal depression is common during the perinatal period [6], and strong support systems may help buffer against various stressors commonly experienced during this often stressful time. For instance, social support has been shown to enhance maternal self-efficacy, increasing confidence in one's ability to successfully perform certain behaviors and take on new roles [48]. Moreover, previous evidence suggests that perceptions of social support dampen physiological stress responses by downregulating sympathetic, hypothalamic-pituitary-adrenal (HPA) axis, and inflammatory reactions to stressors $[9,49,50]$, thereby dampening the harmful effects of perinatal stress and potentially decreasing the risk of PPD.

However, the ongoing COVID-19 pandemic has dramatically impacted everyday life, leading to widespread perceptions of isolation and reduced social support [5153]. These changes may disproportionately impact new mothers. Preliminary research has demonstrated that the COVID-19 pandemic has increased reported feelings of loneliness and poor mental health among mothers, as shelter-in-place orders have disrupted daily life and inperson interactions with others [42, 54]. Additionally, the pandemic has also inhibited access to needed support services like childcare, a trend that appears to most strongly affect mothers (compared to fathers or other caregivers), especially working mothers [23, 24]. Research prior to the pandemic indicates that new mothers who report greater levels of work spillover into the home exhibited lower mental health scores and that assistance in newborn care from family members was a consistent predictor of wellbeing $[11,55]$. It therefore seems likely that the blurring of work-home boundaries during the pandemic has had a negative impact on maternal mental health, while reduced help around the house during the postpartum period and unreliable access to childcare may compound this issue and increase PPD risk.

Interestingly, timing of giving birth in the course of the pandemic was not significantly related to maternal PPD score in either model. It is possible that this lack of an association is due to the pandemic exerting different effects on mental health as the COVID-19 pandemic persists over time. For instance, mothers may experience an increased risk for depression early in pandemic in response to initial disruptions to daily life and COVID19-related feelings of panic and uncertainty. Yet, while the initial negative emotions and social disruptions may have partly subsided over time, elevated maternal depression risk may have persisted as individuals instead suffered from pandemic fatigue and/or financial worries [4, $56,57]$. Future research is needed to assess how specific factors contributing to maternal depression risk during the COVID-19 pandemic may vary over time.

\section{Maternal education level and pandemic-related changes in support}

The risk of poor mental health outcomes, including PPD, is not uniformly experienced across all groups $[9,58,59]$. Previous work suggests that socioeconomic status (SES) is a significant predictor of PPD risk, such that individuals of low SES exhibit the greatest risk of PPD [58, 59]. Socioeconomic status is a summary measure of individual social and economic position in relation to others and is shaped by many factors, including income and education level. Specifically, higher education and income levels are associated with increased SES, and also with lower PPD risk $[58,59]$. Higher SES may help buffer against depression through reducing the stressors mothers face on a daily basis, while also facilitating access to support networks (e.g., hired help for housework, in-home childcare providers, mother-infant activity groups such as baby yoga, etc.) $[60,61]$. However, the benefits associated 
with higher SES with regards to mental health may be diminished during the COVID-19 pandemic.

For example, income level was not associated with increased likelihood of sustained help with household work and newborn care or continued access to childcare. Conversely, education level appeared to be more consistently related with disrupted instrumental support, but not in the expected direction. More highly educated participants were more likely to report that they were receiving less help with housework and newborn care due to the pandemic, and were also more likely to indicate that their access to childcare had been disrupted. One possible explanation for this surprising pattern is that the pandemic has more strongly impacted nonparental instrumental support utilized by well educated, high SES individuals (e.g., hired help and nonparental childcare such as nannies or daycares) [62, 63]. For instance, well educated, high SES parents are likely better able to afford the high costs of non-parental childcare in the U.S., allowing mothers to continue working $[64,65]$. It is also possible that more highly educated individuals rely on these services in part because they are more likely to live farther away from familial support systems $[66,67]$.

Evidence suggests that a positive trend exists between education level and relocation due to work-related reasons (as opposed to family-related or housing-related reasons), with highly educated individuals tending to relocate to areas with more employment opportunities $[66,67]$. In other words, mothers with higher education levels may relocate far from family for work-related reasons more often than individuals with less formal education. While additional work is needed to test this hypothesis, preliminary analyses using the CARE study database has documented a positive association between education level and the likelihood of participants reporting that their loved ones were unable to meet their infant due to the pandemic (unpublished data), suggesting that highly educated participants may live farther from family members who were unable to safely travel during the pandemic to provide support during the postpartum period. As has been documented elsewhere $[68,69]$, it is also possible that more high educated participants in this sample were more likely to comply with recommended preventive measures; thereby decreasing the likelihood of allowing loved ones to visit or using childcare services outside of the home. Future studies should explore how available instrumental support during the pandemic may vary by SES measures, such as education level.

In addition to SES measures, additional work using more diverse samples is required to examine how race/ ethnicity may be associated with employment and instrumental support access during the pandemic. People of color exhibit greater exposure to psychosocial and economic stressors, increasing their PPD risk [9, 59]. Communities of color have also been disproportionately affected by the COVID-19 pandemic, with higher morbidity and mortality rates [70-72]. Additionally, individuals of color are overrepresented among low-wage essential workers; positions which require parents to continue working outside the home during the pandemic, a challenging prospect when childcare services remain closed [73, 74]. It is therefore critical to assess how the ongoing pandemic may affect PPD risk in minority populations and determine how individuals may draw on existing or novel support networks to buffer against pandemic-related stressors. Previous work indicates that enhanced social support may decrease the risk of PPD in people of color [9], suggesting that fostering strong support networks may represent an important non-pharmaceutical strategy to support mental health during the postpartum period across diverse communities. Future work should explore examples of resilience and social support during the COVID-19 pandemic.

\section{Healthcare and policy implications}

Research prior to the COVID-19 pandemic suggests that PPD goes undiagnosed in one of every five to eight postpartum individuals, translating to more than a half a million individuals going undiagnosed each year [10, 75]. This pattern may be due in part to a lack of PPD screening. Results of a multiple-site study (31 sites) in the U.S. revealed that one in eight individuals with a live birth reported not being asked about depression during a postpartum visit [75]. More consistent screening protocols are therefore needed, especially during the COVID-19 pandemic as PPD levels rise, to correctly identify PPD cases in order to initiate appropriate care. In addition to screening more frequently, providers and policymakers should consider which factors may either increase or decrease PPD risk, both during and after the COVID-19 pandemic.

For example, the results presented here cumulatively suggest that medical care providers should consider sustained postpartum instrumental support as a strategy to support maternal mental health during the pandemic. Interventions that enhance support may consequently offer an efficient, non-pharmaceutical technique to protect maternal mental health and reduce depression risk [41], especially if combined with other interventions. Thus, policies designed to reduce disease transmission and allow individuals to safely interact with others -- such as investing in widespread and regular COVID-19 testing -- may reduce disruptions to instrumental support received during the postpartum period and reduce the risk of PPD (i.e., by allowing childcare services to remain safely open and help mothers feel comfortable allowing 
individuals with negative tests to visit and help with household work and newborn care). Yet, any novel PPD interventions during the COVID-19 pandemic should also consider how the pandemic may impact individuals differently, with implications for disrupted support networks and subsequent poor mental health outcomes.

\section{Limitations}

It should be noted that despite the strengths of these analyses (e.g., large sample size and participants from across the U.S.), several important study limitations exist. First, as mentioned above, the study survey did not explicitly ask about in-person school attendance as a form of childcare access, and it is unclear how participants may have reported this type of childcare. Likewise, although we included several relevant confounders in the statistical models, PPD is a complex condition with many possible confounders (e.g., previously experienced trauma and poor health, additional markers of socioeconomic status such as neighborhood disadvantage, etc.). It is consequently likely these models failed to account for all relevant factors that influence PPD risk because these data were not collected in the study survey. Future studies should expand upon these analyses and include additional possible confounders during statistical analysis. In addition, this study is cross-sectional. It is therefore not possible to definitively determine whether the significant relationship observed between reported social support and maternal depression score is due to instrumental support protecting against depression or to maternal depression altering perceptions of received instrumental support. Longitudinal data collection is needed to establish causal relationships. In addition, due to the use of convenience sampling, these data are not representative of the U.S. population as a whole; white, educated, wealthy individuals are overrepresented in the present sample compared to the U.S. birthing population [76]. Additional work is needed to determine whether the associations observed here are also evident across a more representative, diverse sample of the U.S. population.

\section{Conclusions}

The COVID-19 pandemic has significantly disrupted instrumental support systems, including for individuals in the postpartum period. Postpartum recovery is bolstered by assistance from others, via help with household chores, newborn care, and watching other children. However, typical systems of support -- including family, friends, and paid help -- may have been impacted by the pandemic due to social distancing mandates. Our findings indicate that the likelihood of reporting uninterrupted help with housework, newborn care, and childcare for older children in the early postpartum period has changed over time. Respondents who gave birth later in the course of the pandemic were more likely to indicate that the pandemic had not affected the help they received with household work and newborn care, suggesting that participants who gave birth earlier in the pandemic were more likely to experience disruptions to these aspects of social support (potentially due to the more restrictive shelterin-place orders evident at the start of the pandemic). Access to childcare also varied over time, although this relationship was nonlinear and suggested that a higher proportion of participants reported continued access to childcare beginning in late August, perhaps coinciding with the start of the school year and students returning to in-person classroom instruction.

Our results also suggest that social support systems known to protect against PPD may be especially important in supporting mental health during the pandemic, such that respondents who reported that the pandemic had not disrupted instrumental support with household work and newborn care exhibited lower depression scores. Likewise, among a subset of participants with other children, continued access to childcare during the pandemic was associated with lower depression scores. Instrumental support during the postpartum period therefore represents a potential tool to protect against PPD, both during and after the COVID-19 pandemic.

\section{Acknowledgements \\ We would like to thank Nadia Clement for her assistance building the online survey and designing the recruitment flyers. We would also like to thank Margaret Sherin and Chlöe Sweetman for their valuable feedback on the questionnaire during development and Matthew Nagy for his assistance in data coding and graphing. Further, we express our gratitude to Amanda Lu for her assistance with background research. \\ Authors' contributions \\ TEG is a co-PI of the CARE project, conducted the statistical analyses presented here, and drafted most of the manuscript. ZMT is a co-PI of the CARE project and helped to edit the manuscript. GU, RLM, and GCA assisted in drafting the manuscript, provided manuscript feedback, and helped make the tables. All authors read and approved the final manuscript.}

\section{Funding}

Dr. Zaneta Thayer was funded by the Wenner-Gren Hunt Fellowship (grant \#9687) during this project. Participant compensation was provided through the Claire Garber Goodman Fund in the Department of Anthropology at Dartmouth College.

\section{Availability of data and materials}

The datasets used and analyzed during the current study are available from the corresponding author upon reasonable request.

\section{Declarations}

Ethics approval and consent to participate

This study received IRB approval from the Dartmouth College Committee for the Protection of Human Subjects (STUDY00032045) and all research was 
performed in accordance with the Declaration of Helsinki. Study information (e.g., study summary, purpose, benefits, risks, and privacy protection details) was provided to all participants prior their completion of the survey. Informed consent was collected by asking potential participants to click a box saying that they had read the study information and consented to participating in the study.

\section{Consent for publication}

Not applicable.

\section{Competing interests}

The authors declare that they have no competing interests.

\section{Author details}

'Department of Anthropology, Washington University in St. Louis, St. Louis, MO, USA. ${ }^{2}$ Department of Anthropology, Dartmouth College, Hanover, NH, USA. ${ }^{3}$ Society of Fellows, Dartmouth College, Hanover, NH, USA. ${ }^{4}$ Ecology, Evolution, Environment \& Society Program, Dartmouth College, Hanover, NH, USA.

Received: 29 March 2021 Accepted: 15 November 2021

Published online: 13 December 2021

\section{References}

1. Dennis C-L, Fung K, Grigoriadis S, Robinson GE, Romans S, Ross L. Traditional postpartum practices and rituals: a qualitative systematic review. Women's Health. 2007;3(4):487-502.

2. Grigoriadis S, Robinson GE, Fung K, Ross LE, Chee C, Dennis C-L, et al. Traditional postpartum practices and rituals: clinical implications. Can J Psychiatry. 2009;54(12):834-40.

3. Batra K, Pharr J, Olawepo JO, Cruz P. Understanding the multidimensional trajectory of psychosocial maternal risk factors causing preterm birth: a systematic review. Asian J Psychiatr. 2020;54:102436.

4. Thayer ZM, Gildner TE. COVID-19-related financial stress associated with higher likelihood of depression among pregnant women living in the United States. Am J Hum Biol [Internet]. 2020 Sep 22 [cited 2020 Nov 11]; Available from: https://www.ncbi.nlm.nih.gov/pmc/articles/PMC75 36992/

5. Gildner TE, Laugier EJ, Thayer ZM. Exercise routine change is associated with prenatal depression scores during the COVID-19 pandemic among pregnant women across the United States. PLoS One. 2020;15(12):e0243188.

6. Milgrom J, Hirshler Y, Reece J, Holt C, Gemmill AW. Social support-a protective factor for depressed perinatal women? Int J Environ Res Public Health. 2019;16(8):1426.

7. Dennis C-L, Ross L. Women's perceptions of partner support and conflict in the development of postpartum depressive symptoms. J Adv Nurs. 2006;56(6):588-99.

8. Li Y, Long Z, Cao D, Cao F. Social support and depression across the perinatal period: a longitudinal study. J Clin Nurs. 2017;26(17-18):2776-83.

9. Pao C, Guintivano J, Santos H, Meltzer-Brody S. Postpartum depression and social support in a racially and ethnically diverse population of women. Arch Womens Ment Health. 2019;22(1):105-14.

10. Corrigan CP, Kwasky AN, Groh CJ. Social support, postpartum depression, and professional assistance: a survey of mothers in the Midwestern United States. J Perinat Educ. 2015;24(1):48-60.

11. Grice MM, McGovern PM, Alexander BH, Ukestad L, Hellerstedt W. Balancing work and family after childbirth: a longitudinal analysis. Womens Health Issues. 2011;21(1):19-27.

12. Dib S, Rougeaux E, Vázquez-Vázquez A, Wells JCK, Fewtrell M. Maternal mental health and coping during the COVID-19 lockdown in the UK: data from the COVID-19 new mum study. Int J Gynecol Obstet. 2020;151(3):407-14.

13. Stana A, Miller A. "Being a mom = having all the feels": social support in a postpartum depression online support group. Atlantic Journal of Communication. 2019;27:1-14.

14. Keefe RH, Brownstein-Evans C, Rouland Polmanteer RS. Having our say: African-American and Latina mothers provide recommendations to health and mental health providers working with new mothers living with postpartum depression. Soc Work Ment Health. 2016;14(5):497-508.
15. Negron R, Martin A, Almog M, Balbierz A, Howell EA. Social support during the postpartum period: mothers' views on needs, expectations, and mobilization of support. Matern Child Health J. 2013;17(4):616-23.

16. Thoits PA. Mechanisms linking social ties and support to physical and mental health. J Health Soc Behav. 2011;52(2):145-61.

17. Leahy-Warren P, McCarthy G, Corcoran P. Postnatal depression in first-time mothers: prevalence and relationships between functional and structural social support at 6 and 12 weeks postpartum. Arch Psychiatr Nurs. 2011;25(3):174-84

18. Webber E, Benedict J. Postpartum depression: a multi-disciplinary approach to screening, management and breastfeeding support. Arch Psychiatr Nurs. 2019;33(3):284-9.

19. CDC. Depression During and After Pregnancy [Internet]. Centers for Disease Control and Prevention. 2020 [cited 2021 Mar 29]. Available from: https://www.cdc.gov/reproductivehealth/features/maternal-depression/ index.html

20. Singhal $A$. The role of infant nutrition in the global epidemic of noncommunicable disease. Proc Nutr Soc. 2016;75(2):162-8.

21. Reid KM, Taylor MG. Social support, stress, and maternal postpartum depression: a comparison of supportive relationships. Soc Sci Res. 2015;54:246-62.

22. Gildner TE, Thayer ZM. Birth plan alterations among American women in response to COVID-19. Health Expectations [Internet]. 2020 [cited 2020 Jun 9]; Available from: https://onlinelibrary.wiley.com/doi/abs/10.1111/ hex.13077

23. Alon T, Doepke M, Olmstead-Rumsey J, Tertilt M. The Impact of COVID-19 on Gender Equality [Internet]. National Bureau of Economic Research; 2020 Apr [cited 2021 Mar 29]. Report No.: w26947. Available from: https:// www.nber.org/papers/w26947

24. Malkawi SH, Almhdawi K, Jaber AF, Alqatarneh NS. COVID-19 quarantinerelated mental health symptoms and their correlates among mothers: a cross sectional study. Matern Child Health J. 2020:1-11.

25. Liang P, Wang Y, Shi S, Liu Y, Xiong R. Prevalence and factors associated with postpartum depression during the COVID-19 pandemic among women in Guangzhou, China: a cross-sectional study. BMC Psychiatry. 2020;20(1):557.

26. Spinola O, Liotti M, Speranza AM, Tambelli R. Effects of COVID-19 epidemic lockdown on postpartum depressive symptoms in a sample of Italian mothers. 2020.

27. Ritchie H. Google mobility trends: How has the pandemic changed the movement of people around the world. Our World in Data Available online: https://ourworldindata.org/covid-mobility-trends (accessed on 17 August 2020). 2020.

28. Jacobsen GD, Jacobsen KH. Statewide COVID-19 stay-at-home orders and population mobility in the United States. World Medical \& Health Policy. 2020;12(4):347-56.

29. Engle S, Stromme J, Zhou A. Staying at Home: Mobility Effects of COVID19 [Internet]. Rochester, NY: Social Science Research Network; 2020 Apr [cited 2021 Mar 29]. Report No.: ID 3565703. Available from: http://papers. ssrn.com/abstract $=3565703$

30. Harris PA, Taylor R, Thielke R, Payne J, Gonzalez N, Conde JG. Research electronic data capture (REDCap) — a metadata-driven methodology and workflow process for providing translational research informatics support. J Biomed Inform. 2009;42(2):377-81.

31. Harris PA, Taylor R, Minor BL, Elliott V, Fernandez M, O'Neal L, et al. The REDCap consortium: Building an international community of software platform partners. Journal of Biomedical Informatics. 2019;95:103208.

32. Cox J, Holden J, Sagovsky R. Edinburgh postnatal depression scale (EPDS). Br J Psychiatry. 1987;150:782-6.

33. Pace CC, Spittle AJ, Cheong JLY, Davis PG, Doyle LW, Treyvaud K, et al. Evolution of Depression and Anxiety Symptoms in Parents of Very Preterm Infants During the Newborn Period 2016;8.

34. Wisner KL, Chambers C, Sit DKY. Postpartum depression: a major public health problem. JAMA. 2006;296(21):2616.

35. McCue Horwitz S, Briggs-Gowan MJ, Storfer-Isser A, Carter AS. Prevalence, correlates, and persistence of maternal depression. J Women's Health. 2007 Jun;16(5):678-91.

36. Office of Management and Budget Standards. Revisions to the Standards for the Classification of Federal Data on Race and Ethnicity [Internet]. 1997. Available from: https://www.govinfo.gov/content/pkg/FR-1997-1030/pdf/97-28653.pdf 
37. Morris-Rush JK, Freda MC, Bernstein PS. Screening for postpartum depression in an inner-city population. Am J Obstet Gynecol. 2003;188(5):1217-9.

38. Matthey S, Henshaw C, Elliott S, Barnett B. Variability in use of cut-off scores and formats on the Edinburgh postnatal depression scale implications for clinical and research practice. Arch Womens Ment Health. 2006;9(6):309-15.

39. Svetunkov I, Petropoulos F. Old dog, new tricks: a modelling view of simple moving averages. Int J Prod Res. 2018;56(18):6034-47.

40. Nielsen D, Videbech P, Hedegaard M, Dalby J, Secher NJ. Postpartum depression: identification of women at risk. BJOG Int J Obstet Gynaecol. 2000;107(10):1210-7.

41. Wang L, Wu T, Anderson JL, Florence JE. Prevalence and risk factors of maternal depression during the first three years of child rearing. J Women's Health. 2011;20(5):711-8.

42. Davenport MH, Meyer S, Meah VL, Strynadka MC, Khurana R. Moms Are Not OK: COVID-19 and Maternal Mental Health. Front Glob Womens Health [Internet]. 2020 [cited 2020 Jun 23];1. Available from: https://www. frontiersin.org/articles/10.3389/fgwh.2020.00001/full

43. Chen H, Selix N, Nosek M. Perinatal Anxiety and Depression During COVID-19. The Journal for Nurse Practitioners [Internet]. 2020 Sep 30 [cited 2020 Dec 8]; Available from: http://www.sciencedirect.com/scien ce/article/pii/S1555415520305316

44. World Health Organization. Pandemic fatigue: reinvigorating the public to prevent COVID-19: policy framework for supporting pandemic prevention and management: revised version November 2020. World Health Organization. Regional Office for Europe; 2020.

45. Chiu A. A pandemic pod could help you get through winter, experts say. Here's how to form one. Washington Post [Internet]. 2020 [cited 2020 Dec 5]; Available from: https://www.washingtonpost.com/lifestyle/wellness/ pandemic-pod-winter-covid/2020/10/14/214ed65c-0d63-11eb-b1e816b59b92b36d_story.html

46. Moyer MW. The Dos and Don'ts of 'Quarantine Pods.'The New York Times [Internet]. 2020 Jun 9 [cited 2020 Dec 5]; Available from: https://www. nytimes.com/2020/06/09/parenting/coronavirus-pod-family.html

47. Gan Y, Xiong R, Song J, Xiong X, Yu F, Gao W, et al. The effect of perceived social support during early pregnancy on depressive symptoms at 6 weeks postpartum: a prospective study. BMC Psychiatry. 2019;19(1):232.

48. Vaezi A, Soojoodi F, Banihashemi AT, Nojomi M. The association between social support and postpartum depression in women: a cross sectional study. Women and Birth. 2019;32(2):e238-42.

49. Dickerson SS, Zoccola PM. Toward a biology of social support. In: Oxford handbook of positive psychology, 2nd ed. New York, NY, US: Oxford University Press; 2009. p. 519-26. (Oxford library of psychology).

50. Uchino N, Cacioppo JT, Kiecolt-Glaser JK. The Relationship Between Social Support and Physiological Processes: A Review With Emphasis on Underlying Mechanisms and Implications for Health 1996;44.

51. Banerjee D, Rai M. Social isolation in Covid-19: the impact of loneliness. Int J Soc Psychiatry. 2020;66(6):525-7.

52. Pietrabissa G, Simpson SG. Psychological Consequences of Social Isolation During COVID-19 Outbreak. Front Psychol [Internet]. 2020 [cited $2021 \mathrm{Jan}$ 7];11. Available from: https://www.frontiersin.org/articles/10. 3389/fpsyg.2020.02201/full

53. Usher K, Bhullar N, Jackson D. Life in the pandemic: social isolation and mental health. J Clin Nurs. 2020;29(15-16):2756-7.

54. Hessami K, Romanelli C, Chiurazzi M, Cozzolino M. COVID-19 pandemic and maternal mental health: a systematic review and meta-analysis. J Matern Fetal Neonatal Med. 2020:1-8.

55. Ozer EM. The impact of childcare responsibility and self-efficacy on the psychological health of professional working mothers. Psychol Women Q. 1995;19(3):315-35.

56. Li D, Chaudhary H, Zhang Z. Modeling spatiotemporal pattern of depressive symptoms caused by COVID-19 using social media data mining. Int J Environ Res Public Health. 2020;17(14):4988.

57. Rolland JS. COVID-19 pandemic: applying a multisystemic Lens. Fam Process. 2020:59(3):922-36.

58. Guintivano J, ManuckT, Meltzer-Brody S. Predictors of postpartum depression: a comprehensive review of the last decade of evidence. Clin Obstet Gynecol. 2018;61(3):591-603.
59. Norhayati MN, Nik Hazlina NH, Asrenee AR, Wan Emilin WMA. Magnitude and risk factors for postpartum symptoms: a literature review. J Affect Disord. 2015;175:34-52

60. Goyal D, Gay C, Lee KA. How much does low socioeconomic status increase the risk of prenatal and postpartum depressive symptoms in first-time mothers? Womens Health Issues. 2010;20(2):96-104.

61. Silverman ME, Burgos L, Rodriguez ZI, Afzal O, Kalishman A, Callipari F, et al. Postpartum mood among universally screened high and low socioeconomic status patients during COVID-19 social restrictions in new York City. Sci Rep. 2020;10(1):22380.

62. Fram MS, Kim J. Race/ethnicity and the start of child care: a multi-level analysis of factors influencing first child care experiences. Early Child Res Q. 2008;23(4):575-90.

63. Huston AC, Chang YE, Gennetian L. Family and individual predictors of child care use by low-income families in different policy contexts. Early Child Res Q. 2002;17(4):441-69.

64. Ruppanner L, Moller S, Sayer L. Expensive childcare and short school days $=$ lower maternal employment and more time in childcare? Evidence from the American Time Use Survey Socius. 2019;5:2378023119860277.

65. Tang S, Coley RL, Votruba-Drzal E. Low-income families' selection of child care for their young children. Child Youth Serv Rev. 2012;34(10):2002-11.

66. Chen Y, Rosenthal SS. Local amenities and life-cycle migration: do people move for jobs or fun? J Urban Econ. 2008;64(3):519-37.

67. Schachter J. Why People Move: Exploring the March 2000 Current Population Survey. U.S. Department of Commerce, Economics and Statistics Administration, Bureau of the Census; 2001. 16 p.

68. Roma P, Monaro M, Muzi L, Colasanti M, Ricci E, Biondi S, et al. How to improve compliance with protective health measures during the covid-19 outbreak: testing a moderated mediation model and machine learning algorithms. Int J Environ Res Public Health. 2020;17(19):7252.

69. Wang D, Marmo-Roman S, Krase K, Phanord L. Compliance with preventative measures during the COVID-19 pandemic in the USA and Canada: results from an online survey. Soc Work Health Care 2021;0(0):1-16.

70. Chowkwanyun M, Reed AL. Racial health disparities and Covid-19 - caution and context. N Engl J Med. 2020;383(3):201-3.

71. Fortuna LR, Tolou-Shams M, Robles-Ramamurthy B, Porche MV. Inequity and the disproportionate impact of COVID-19 on communities of color in the United States: The need for a trauma-informed social justice response. Psychological Trauma: Theory, Research, Practice, and Policy. 2020;12(5):443.

72. Hooper MW, Nápoles AM, Pérez-Stable EJ. COVID-19 and racial/ethnic disparities. Jama. 2020.

73. Bahn K, Cohen J, Rodgers Y. A feminist perspective on COVID-19 and the value of care work globally. Gender Work and Organization. 2020;27:695-9.

74. Fortier N. COVID-19, gender inequality, and the responsibility of the state. International Journal of Wellbeing. 2020;10(3).

75. Bauman BL, Ko JY, Cox S, D'Angelo MPHDV, Warner L, Folger S, et al. Vital signs: postpartum depressive symptoms and provider discussions about perinatal depression - United States, 2018. MMWR Morb Mortal Wkly Rep. 2020;69(19):575-81.

76. Martin JA, Hamilton BE, Osterman MJ, Driscoll AK. Births: Final data for 2018. National vital statistics reports [Internet]. 2019;68(13). Available from: https://stacks.cdc.gov/view/cdc/82909

\section{Publisher's Note}

Springer Nature remains neutral with regard to jurisdictional claims in published maps and institutional affiliations. 